Recherches en didactique des langues et des cultures

Les cahiers de l'Acedle

$15-3 \mid 2018$

Quelles médiations en didactique des langues et des cultures?

\title{
Pratiques transfictionnelles en classe de langue à l'école primaire
}

L'album comme support médiateur

Marie-France Burgain

\section{(Q) OpenEdition}

\section{Journals}

Édition électronique

URL : http://journals.openedition.org/rdlc/3644

DOI : $10.4000 /$ rdlc.3644

ISSN : $1958-5772$

Éditeur

ACEDLE

\section{Référence électronique}

Marie-France Burgain, «Pratiques transfictionnelles en classe de lanque à l'école primaire »,

Recherches en didactique des langues et des cultures [En ligne], 15-3 | 2018, mis en ligne le 01

septembre 2018, consulté le 01 mai 2019. URL : http://journals.openedition.org/rdlc/3644 ; DOI :

$10.4000 /$ rdlc.3644

Ce document a été généré automatiquement le 1 mai 2019.

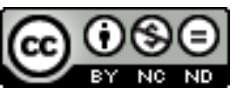

Recherches en didactique des langues et des cultures is licensed under a Creative Commons AttributionNonCommercial-NoDerivatives 4.0 International License 


\section{Pratiques transfictionnelles en classe de langue à l'école primaire}

L'album comme support médiateur

Marie-France Burgain

\section{Introduction}

1 L'album de littérature de jeunesse, «forme d'expression présentant une interaction de textes (qui peuvent être sous-jacents) et d'images (spatialement prépondérantes) au sein d'un support " (Van der Linden, 2006: 87) a toute sa place à l'école maternelle et élémentaire et constitue une entrée privilégiée dans l'écrit et la lecture en $\mathrm{L}^{1}{ }^{1}$. De nombreux ouvrages, figurant dans les listes officielles du ministère, sont des textes écrits à l'origine dans une langue étrangère, spécificité qui n'apparaît cependant pas dans l'exploitation que l'on en propose; en conséquence, "l'œuvre littéraire perd sa dimension culturelle.» (Deyrich, 2011: 206). L'album est donc également considéré comme un support intéressant pour l'enseignement des langues vivantes à l'école primaire car, contrairement à ce qui se fait avec les textes traduits, «l'intégration d'ceuvres de littérature de jeunesse étrangère dans le cours de L2 peut ainsi donner l'occasion de relier et langues et cultures à partir d'un support totalement authentique. » (Deyrich, 2011 : 206).

Des ouvrages pédagogiques, dans la lignée de ceux de Ellis et Brewster (2007), tel Enseigner l'anglais avec des albums d'Anthony Browne (Choquet et Thieffry, 2012) ou Découvrir l'anglais avec des albums de jeunesse des mêmes auteurs (2013), proposent des pistes d'exploitation des albums en langue étrangère. L'album apparaît ainsi comme une entrée dans la langue et la culture étrangères à privilégier, véritable support "médiateur", au sens d'intermédiaire mais aussi de "facilitateur " entre l'élève et de nouveaux savoirs. Son exploitation permet à l'élève d'acquérir des connaissances grammaticales, lexicales et phonologiques tout en construisant une compétence interculturelle. Les choix stylistiques des auteurs et les illustrations contribuent, en outre, à faciliter le travail de compréhension et de mémorisation du texte. Pour citer à nouveau Deyrich : 
L'utilisation de l'album est de plus en plus fréquente pour la L2, en raison du point d'appui que représente l'articulation entre texte et image et des possibilités offertes pour la médiation dans le cours (Deyrich, 2011 : 211).

La question que pose alors Ouvrard est celle de l'articulation entre compréhension et interprétation :

Pourrait-on dire alors que l'exploitation d'un album authentique en classe de langue rejoint celle de la littérature de jeunesse en français et qu'interpréter le sens $\mathrm{du}$ texte, s'atteler aux problèmes que pose le texte constitue également un objectif essentiel ? (Ouvrard, $2013: 66$ ).

Est-il possible en L2 à l'école primaire d'exploiter l'album de littérature de jeunesse autrement que comme un simple support prétexte à la découverte de faits linguistiques ou culturels? En d'autres termes, son utilisation en langue étrangère permet-elle également aux élèves de développer des compétences de lecteurs? L'hypothèse est que le travail d'interprétation se fait grâce à l'appropriation de l'histoire par l'élève et au passage à l'expression orale et/ou écrite. La recherche en littérature, et plus particulièrement les travaux de Saint-Gelais (1999) sur la transfictionnalité, nous permettront d'avoir quelques éléments de réponse.

Enfin, la dernière question qui se pose est celle de l'articulation entre exploitation du texte littéraire et perspective actionnelle : existe-t-il des "tâches littéraires » (Luscher, 2009) ? Celles-ci ne permettraient-elles pas alors d'associer texte littéraire et agir social et de donner la possibilité à l'élève à son tour de transmettre et de devenir médiateur?

Pour répondre à toutes ces questions, le premier point portera sur ce que disent les programmes de 2015 sur l'utilisation des albums de jeunesse en cours de langue. Puis, un détour par la recherche en littérature nous permettra de revenir sur la définition et les enjeux des pratiques transfictionnelles. Enfin, il s'agira de voir comment ces pratiques peuvent devenir des tâches finales littéraires à l'école primaire, permettant à l'élève de construire des compétences de lecteur et de devenir à son tour créateur et médiateur.

\section{L'album comme support médiateur}

\section{L'album, support d'acquisitions linguistiques}

6 Les ouvrages de littérature de jeunesse, et plus particulièrement les albums, occupent une place privilégiée dans les programmes de langues de 2015. L'entrée culturelle « l'univers enfantin " pour le cycle 2 comprend « les monstres, fées et autres références culturelles de la littérature enfantine » et la «littérature enfantine » elle-même et, celle intitulée «l'imaginaire » pour le cycle 3, «la littérature de jeunesse, œuvres célèbres et grands récits populaires » et « les héros et personnages de fiction ».

7 L'utilisation de ces textes en version originale permet à l'élève d'acquérir des connaissances lexicales, grammaticales et phonologiques en langue étrangère. Ces ouvrages proposent des structures grammaticales simples et des champs lexicaux correspondant aux programmes officiels de langues, qui s'articulent autour de l'enfant et de son environnement immédiat au cycle 2, domaine qui s'élargit un peu au cycle 3 . Les albums apparaissent alors comme des supports médiateurs permettant d'apprendre la langue et de découvrir la culture des pays concernés².

8 Les choix stylistiques des auteurs (effets de répétition ou d'accumulation, jeu sur les rimes par exemple) proposent également des exemples des spécificités orales de la 
langue ; le recours aux illustrations permet de mettre en place un autre jeu avec le lecteur tout en facilitant son travail de compréhension et de mémorisation. Autant de qualités propres à ces ouvrages qui en font des outils adaptés à l'enseignement d'une langue.

Il n'est pas non plus étonnant de retrouver les albums dans les exemples de connaissances et compétences associées aux activités langagières de communication proposées par les programmes : y figurent «suivre le fil d'une histoire très courte » au cycle 2 et « suivre le fil d'une histoire simple " au cycle 3 en compréhension orale. "Raconter une histoire courte à partir d'images ou de modèles déjà rencontrés » au cycle 2 et "raconter une histoire courte à l'aide de supports visuels » au cycle 3 apparaissent en prise de parole en continu. Le "texte de fiction" apparaît également dans les supports conseillés pour travailler la compréhension écrite et « raconter succinctement des expériences vécues ou imaginées » est une des activités proposées en expression écrite.

De plus, ce support propose des personnages et des situations qui facilitent l'identification du lecteur et l'investissement des élèves. Les personnages principaux y sont des enfants ou des animaux aux traits de caractère semblables à ceux des enfants eux-mêmes. Ces qualités justifient le recours aux textes littéraires en classe de langue à l'école primaire, comme le dit Galani (2009: 264) : «[En particulier,] l'exploitation didactique du texte littéraire en classe [de FLE] est justifiée par trois raisons: des raisons linguistiques, culturelles et d'épanouissement affectif.»

\subsection{L'album, médiateur culturel}

11 En cours de L2 à l'école primaire, les élèves doivent découvrir "les éléments culturels en contexte grâce aux possibilités offertes par la vie de classe » (Programmes de cycle 2:31) et la lecture d'un album de littérature de jeunesse fait partie de ces possibilités. La lecture d'albums en L2 permet à l'élève de découvrir un objet culturel authentique. Il s'agit d'une véritable prise de conscience interculturelle :
La littérature constitue un point d'appui dans le développement de l'enfant : il a besoin d'histoires, de récits pour pouvoir se signifier à lui-même la structure du monde, la place qu'il y occupe et celle de l'autre. La découverte de l'univers de l'autre dans la L2, dans des ouvrages destinés au public de cet âge permet de vivre une expérience authentique de l'altérité. Il est ainsi amené à vivre une lecture dans la L2 dans un univers littéraire propre à l'enfance, une lecture susceptible d'ailleurs d'être menée par des enfants natifs de la L2, de leur point de vue. En résumé, l'altérité correspond alors à une découverte et à un partage d'un univers enfantin à partir de supports littéraires en version originale et, partant, à une prise en considération des synergies et des différences linguistiques et culturelles (Deyrich, $2011: 210-211)$.

Parce qu'il présente aux élèves une histoire écrite et inscrite dans une langue et une culture différentes des leurs, l'album leur permet de construire une compétence interculturelle. La littérature de jeunesse a ainsi, selon Morel (2012:147), « une fonction de médiateur/ médiatrice au sens défini par Geneviève Zarate" qui décrit le médiateur comme celui qui « s'autorise à intervenir dans les processus qui dramatisent les ruptures de sens entre soi et les autres et s'emploie à rétablir la continuité » (Zarate, $2003: 61$ ).

Est-il alors possible de dépasser le stade de l'utilisation de l'album comme support ou prétexte à l'acquisition de connaissances linguistiques et à la découverte d'une autre culture? L'enseignant peut-il proposer aux élèves un travail qui leur permettra aussi de développer des compétences littéraires? 


\section{Pratiques transfictionnelles en littérature de jeunesse}

\subsection{Transfictionnalité : définition et enjeux}

14 Une tendance de plus en plus forte en littérature, et tout particulièrement en littérature de jeunesse actuellement, est la multiplication des pratiques transfictionnelles. La transfictionnalité, telle que définie par Saint-Gelais (2011), se caractérise par le partage d'un univers de fiction et la migration de données diégétiques d'un texte vers un autre :

Par «transfictionnalité ", j'entends le phénonème par lequel au moins deux textes, du même auteur ou non, se rapportent conjointement à une même fiction, que ce soit par reprise de personnages, prolongement d'une intrigue préalable ou partage d'univers fictionnel (Saint-Gelais, $2011: 7$ ).

L'idée de départ de Saint-Gelais (2012) lui est venue en observant les "prolongements tentaculaires sur plusieurs médias » auxquels la série Star Trek a donné lieu et surtout la "participation des fans eux-mêmes à cette production fictionnelle apparemment inépuisable autour d'un univers fictif » (Saint-Gelais, 2012:1). Le mot «texte » s'entend au sens large et peut être un récit écrit ou filmique ou télévisuel. L'ensemble des œuvres s'articule autour de la notion de monde et non de récit. Ces prolongements ou réécritures peuvent être proposés par les auteurs eux-mêmes ${ }^{3}$ et par d'autres personnes, les lecteurs, ajoutant des éléments fictifs mais suscitant de nouvelles incomplétudes.

Bien évidemment, pour écrire, il faut d'abord avoir lu; les actes de lecture et d'écriture sont indissociables ${ }^{4}$ : prolongement d'un récit, changement du point de vue sur l'histoire ou nouvelle aventure d'un protagoniste en sont des illustrations. Les opérations en jeu décrites par Saint-Gelais (2011) sont l'expansion (où l'auteur enrichit certains pans de l'univers diégétique), la version (qui propose une perspective ou une intrigue différente), le croisement (où des éléments tirés de plusieurs textes s'entrecroisent) et la capture (reprise de personnages ou d'éléments fictifs dans un cadre différent). Le texte cyclique ou sériel surtout, par son incomplétude, encourage le lecteur à anticiper la suite et à combler les blancs de l'histoire, portraits inachevés de protagonistes ou mystères non résolus. Il s'agit bien de meubler la période de vide entre la publication de deux tomes par exemple en échafaudant des hypothèses s'appuyant sur une solide connaissance de l'univers diégétique.

De très nombreux éléments fictifs restent indéterminés dans une œuvre littéraire, encourageant «le fourmillant et silencieux travail de complétion auquel se livrent les lecteurs de fiction» (Saint-Gelais, 1999: 9). L'écriture transfictionnelle semble alors répondre à un besoin du lecteur. Ces «fanfictions » sont des espaces transitionnels, tout comme le doudou est l'objet transitionnel qui remplace la mère : elles servent à combler une absence, quand le texte est incomplet, ou à compenser la séparation, quand il s'agit d'attendre le prochain tome. L'investissement émotionnel et affectif des lecteurs y est très fort :

La transfictionnalité peut aussi se comprendre comme un miroir qui se promène devant l'imaginaire des lecteurs qui y projettent leur propre structure de fiction (Carrier-Lafleur, 2012 : 472).

Ces «fanfictions » révèlent la façon dont les fans interprètent et s'approprient l'œuvre source. Tout en s'inspirant de l'univers fictionnel d'origine, ils y racontent ce qui, à leurs yeux, manque à ces textes: les écrits de ces récepteurs-rédacteurs ont une dimension méta-textuelle. 
La dimension de partage est étroitement associée à l'écriture de "fanfictions ». Elles n'existent que parce qu'elles sont soumises au jugement d'autrui. Collecter, classer, corriger ou améliorer les récits sont les activités premières de ces sites de fans qui offrent également des espaces de partage et de discussion. L'élaboration des textes elle-même se fait souvent de manière collective, établissant une collaboration concrète entre différents partenaires. Les fans ont, en outre, diffusé leurs productions sur de nombreux supports médiatiques; l'utilisation des technologies numériques a grandement facilité la transmission de ces textes et a entrainé un développement des récits transfictionnels.

\subsection{Albums et pratiques transfictionnelles}

19 Avec leurs personnages récurrents et leur structure répétitive, les albums se prêtent bien également à ce jeu. Et les auteurs sont les premiers à se livrer à l'exercice des pratiques transfictionnelles quand ils proposent des suites sous la forme d'un prolongement de l'histoire, de la présentation de nouvelles aventures des protagonistes ou tout simplement de la déclinaison de la même, ou quasiment, histoire dans des situations différentes : Meg ${ }^{5}$ fete Halloween, mais aussi Noël et Pâques, va chez le docteur, part en vacances et va même sur la lune et Spot $^{6}$ fête Noël, va au zoo et a aussi sa propre voiture. Typiques de la littérature sérielle, les titres de ces ouvrages reprennent tous le nom du protagoniste auquel est associé un élément de la nouvelle intrigue. Bill Martin Jr et Eric Carle choisissent de décliner plutôt le même univers dans des albums différents ${ }^{7}$ et Anthony Browne joue des réécritures en racontant la même histoire de quatre points de vue différents dans Voices in the Park.

D'autres livres sont un encouragement pour le lecteur à poursuivre le récit ou à enrichir le texte. Les chasseurs malheureux de Shh! We have a Plan de Chris Haughton sont prêts à se remettre en quête d'un nouveau gibier à la dernière page de l'album, qui fonctionne comme une ouverture sur une autre histoire. Des textes comme Things I like d'Anthony Browne, dressant la liste de ce que le narrateur-personnage aime faire, pourraient être prolongés à l'envi.

21 Ne pouvons-nous pas jouer en classe sur les relations entre œuvre et lecteurs? N'est-il pas possible d'associer plaisir du lecteur devenu créateur et apprentissages de l'élève ? $\mathrm{Ne}$ pouvons-nous pas nous appuyer sur ces qualités des textes littéraires et l'investissement des lecteurs/élèves en L2 pour, à travers des pratiques transfictionnelles, combiner apprentissage d'une langue et formation littéraire et faire d'eux de nouveaux médiateurs?

\section{Littérature de jeunesse et perspective actionnelle}

22 La question de l'articulation entre littérature et enseignement/apprentissage des langues a souvent été posée pour l'enseignement des langues vivantes étrangères dans le second degré. Dans le cadre de la perspective actionnelle, préconisée par le Cadre Européen Commun de Référence pour les Langues (CECRL) et les programmes officiels de langues, les séquences d'enseignement construites par l'enseignant doivent déboucher sur la réalisation d'une tâche finale. Des chercheurs, comme Luscher (2009) et Puren (2006, 2012), se sont interrogés sur la place de l'enseignement de la littérature dans cette 
approche actionnelle et sur ce que pourrait être une «tâche littéraire ». Le CECRL indique que :

L'utilisation de la langue pour le rêve ou pour le plaisir est importante au plan éducatif mais aussi en tant que telle. Les activités esthétiques peuvent relever de la production, de la réception, de l'interaction ou de la médiation et être orales ou écrites. Elles comprennent des activités comme [...]

- la réécriture et le récit répétitif d'histoires, etc.

- l'audition, la lecture, l'écriture ou le récit oral de textes d'imagination (bouts rimés, etc.) parmi lesquels des caricatures, des bandes dessinées, des histoires en images, des romans photos, etc.

- le théâtre (écrit ou improvisé)

- la production, la réception et la représentation de textes littéraires [....]

(Conseil de l'Europe, $2001: 47$ )

À l'école primaire, il s'agit de mettre en place un projet qui va déboucher sur une réalisation concrète et qui va permettre "d'articuler les activités langagières tout en faisant sens pour l'apprenant » (Deyrich, 2011 : 209). Les programmes de cycle 3 proposent, comme exemples de situations, d'activités et de ressources pour l'élève de "mobiliser ses acquis langagiers et culturels pour produire des phrases ou un texte personnel en s'appuyant sur une trame connue (d'un message, d'une lettre, d'un poème, de textes informatif, narratif...) ». La littérature de jeunesse semble bien avoir ici toute sa place.

Les théories de la lecture et de la réception en littérature indiquent que l'œuvre littéraire ne prend vraiment tout son sens que dans l'acte de lecture. Le livre est porteur de potentialités, conscientes ou inconscientes pour l'écrivain, que le lecteur va actualiser : " Le sens, loin d'être immanent, se présente comme le résultat d'une rencontre : celle du livre et du lecteur.» (Jouve, 1993: 73). La lecture d'une œuvre est ainsi à la croisée de deux expériences, et la relation à un texte est nécessairement dialogique. Le lecteur est actif et créatif : il coopère et participe à la construction du sens du livre. Les textes proposent au lecteur deux types de jeux, le jeu d'identification ou jeu de rôle (correspondant à l'anglais "playing»), favorisant le transfert imaginaire, et le jeu de décodage ou déchiffrage (" game » en anglais), incitant le lecteur à une certaine prise de distance.

En plaçant le lecteur au centre de la construction du sens d'une œuvre, ces théories nous donnent des indications sur la façon dont la littérature de jeunesse pourrait être exploitée en cours de L2 :

[Des textes de telle sorte] [Les textes littéraires] sont en général considérés comme plus motivants et plus ludiques que les textes ordinaires utilisés en classe de langue. Un apprenant qui travaille avec des textes littéraires, apprend à lire de façon critique, empathique et créative (Galani, 2009: 264).

C'est cet investissement qui va inciter le lecteur à créer et produire :

[La littérature] elle entraîne l'élément affectif et émotionnel ; pour cette raison, elle constitue le moyen privilégié qui incite les apprenants à s'impliquer personnellement dans leur apprentissage (Galani, 2009 : 264).

L'enfant va s'approprier des formules, une structure qu'il va chercher à reproduire dans une reformulation de l'histoire :

On considère en effet, nous dit Anne-Sophie Morel, qu'une lecture active mène à l'écriture. Il s'agit à partir d'un hypotexte, de faire mettre en évidence un procédé d'écriture déclencheur qui permettra l'écriture en écho (Morel, 2012 : 145).

Nous retrouvons bien là les phénomènes à l'œuvre dans l'écriture de «fanfictions » dans lesquelles les lecteurs/auteurs intègrent des éléments ou reprennent des motifs de l'histoire d'origine. 
ment ces activités de "réécriture" s'inscrivent-elles dans une perspective actionnelle? Elles peuvent prendre plusieurs formes et sont bien des tâches littéraires qui permettent à l'élève de devenir acteur :

Considérer que l'écriture peut aider à une meilleure compréhension en lecture, c'est considérer que l'écriture met l'élève en activité ; il se trouve ainsi en position d'utiliser la langue, alors même qu'il est en situation d'apprendre. Pourquoi ? C'est que tout texte écrit est donné, fût-ce en L2, alors que, lorsqu'il est produit en classe, il est construit par l'enfant qui, ce faisant, s'approprie le matériau avec lequel il travaille : la langue (Deyrich et Olive, 2009 : 71).

L'album est alors intermédiaire, médiateur permettant à l'élève de passer d'une forme d'expression à une autre, de s'approprier une langue et des procédés d'écriture pour devenir à son tour créateur. Ajouter d'autres animaux à ceux de Brown Bear, prolongeant ainsi le jeu de questions-réponses, intégrer de nouvelles pages à l'album Things I like d'Anthony Browne, imaginer la venue de Spot à l'école ou sa rencontre avec Meg sont des pratiques transfictionnelles qui pourraient être des tâches finales littéraires.

Certes, ces projets sont des projets pédagogiques, des «tâches scolaires sur le texte " plus que des « actions sociales par les textes », pour reprendre la distinction de Puren (2012), mais ils relèvent bien de la perspective actionnelle. La dimension sociale n'en est pas oubliée : la production finale peut être montrée, lue ou jouée devant les autres enfants de la classe, ceux d'une autre classe voire d'une autre école. Dans le projet qu'elle présente, Deyrich (2011 : 209) nous explique que l'album écrit grâce à une dictée à l'adulte "vient compléter le réseau mais ne le termine pas pour autant : il est ensuite lu à d'autres enfants dans d'autres classes ». Il faut que ces activités restent ludiques et demeurent un plaisir : plaisir de découvrir mais aussi plaisir de produire et de partager.

Les Technologies de l'Information et de la Communication pour l'Enseignement (TICE) vont ainsi, trouver toute leur place dans le dispositif : l'enregistrement des productions des élèves et leur insertion dans un diaporama ou un livre numérique remplacera la dictée à l'adulte. Comme pour les «fanfictions", elles pourront aussi servir à diffuser et partager la production. Comme nous l'explique Morel :

Ces productions écrites collectives à dimension sociale effective, de recueils de nouvelles ou de poèmes, peuvent être publiées sur Internet, qui permet désormais d'assurer un élargissement des collaborations au-delà des seuls élèves de la classe et la diffusion des productions au moyen de la publication sur le Web. Là aussi, la publication finale sur Internet donne désormais potentiellement à toute production des apprenants, une certaine dimension de « réalité sociale » qui peut certainement aider à leur motivation, dans la mesure où c'est l'image qu'ils ont et qu'ils donnent d'eux-mêmes en dehors de l'espace scolaire qui se trouve ainsi mise en jeu (Morel, $2012: 145)$.

Enfin, de transfictionnelles, ces productions peuvent devenir transmédiatiques quand elles sont réalisées sur de nouveaux supports : l'univers de fiction se décline alors sur des médias variés. Les projets permettent de croiser les disciplines, comme évoqué dans les nouveaux programmes.

Parmi les activités de médiations orale et écrite proposées par le CECRL, on retrouve bien sûr l'interprétation et la traduction mais aussi la production de pancartes, d'affichettes commentées et la reformulation: Ces productions transfictionnelles voire transmédiatiques ne relèveraient-elles pas alors d'une forme de médiation entre l'œuvre d'origine et le destinataire de ces nouvelles productions, l'apprenant étant alors devenu lui-même médiateur? 


\section{Conclusion}

3 réécriture de l'album Brown Bear, Brown Bear, What Do You See, intitulé Blue Elephant, élaboré par une classe de grande section de maternelle et mis en ligne sur le site de l'inspection académique de clermont ${ }^{8}$. Cette production révèle des connaissances grammaticales, lexicales, phonologiques et une appropriation d'un objet littéraire et du jeu mis en place avec le lecteur. L'histoire produite intègre de nouveaux éléments lexicaux dans des structures de phrases semblables à celles de l'album et la mise en scène/mise en voix de l'album joue sur l'interaction, forme de réappropriation de l'histoire par les élèves. Ce projet a permis de croiser des disciplines, anglais et arts visuels, et d'utiliser les TICE non seulement pour produire mais aussi pour partager.

Pour citer une dernière fois Morel :

la littérature est un outil de médiation, d'accès à la découverte de l'autre et de soi : elle offre des expériences propres à renforcer la conscience subjective des apprenants, en tant qu'apprenants d'une langue étrangère, mais aussi, plus largement, en tant qu'autres (Morel, $2012: 145$ ).

Grâce à la littérature de jeunesse et aux projets mis en œuvre en classe de L2, les élèves peuvent construire des compétences de lecteurs et devenir à leur tour créateurs et médiateurs, proposant aux autres leur propre version d'une histoire et beaucoup d'euxmêmes.

\section{BIBLIOGRAPHIE}

Carrier-Lafleur, T. (2012). Compte-rendu de Saint-Gelais, R. (2011). Fictions transfuges. La transfictionnalité et ses enjeux. Paris : Editions du Seuil, collection « Poétique ». In analyses [En ligne], volume $7 \mathrm{n}^{\circ}$ 3. pp. 461-481. https://uottawa.scholarsportal.info/ojs/index.php/revueanalyses/issue/view/156.

Choquet, C. \& Thieffry, A. (2012). In Belloque P. (coord.). Enseigner l'anglais avec des albums d'Anthony Browne. Lille : SCÉRÉN-CRDP Nord-Pas-de-Calais, Collection « Outils pour les cycles ».

Choquet, C. \& Thieffry, A. (2013). In Belloque, P. (coord.). Découvrir l'anglais avec des albums de jeunesse. Lille : SCÉRÉN-CRDP Nord-Pas-de-Calais, Collection « Outils pour les cycles ».

Conseil de l'Europe. (2001). Cadre Européen Commun de Référence pour les Langues. Paris : Didier.

Deyrich, M.-C. \& Olive, S. (2009). « Des passerelles entre les apprentissages langagiers et culturels à l'école : du français à l'anglais et réciproquement ». In Forlot, G. (dir.). L'anglais et le plurilinguisme. Pour une didactique des contacts et des passerelles linguistiques. Paris : L'Harmattan (coll. Espaces Discursifs). pp. 59-77.

Deyrich, M.-C. (2011). « L'œuvre littéraire pour articuler les apprentissages en L1 et L2 à l'école primaire ». In Aden, J., Grimshaw, T. \& Penz, H. (dir./eds.). Enseigner les langues-cultures à l'ère de la complexité - Teaching Language and Culture in an Era of Complexity - Approches interdisciplinaires pour 
un monde en reliance - Interdisciplinary Approaches for an Interrelated World -. Bruxelles : Peter Lang, Collection : GRAMM-R. pp. 205-214.

Ellis, G. \& Brewster, J. (2007). Tell it Again! The Storytelling Handbook for Primary English Language Teachers. Londres : Pearson.

Galani, M.-E. (2009). « Privilégier le texte littéraire en classe de FLE ». In Tabaki-Iona, F., Proscolli, A. \& Forakis, K. (dir.). Actes du colloque « La place de la littérature dans l'enseignement du FLE». Athènes : Université Nationale et Capodistrienne d'Athènes. pp. 261-271.

Jouve, V. (1993). La lecture. Paris : Hachette (Collection « Contours littéraires »).

Luscher, Jean-Marc (2009). «L'enseignement de la littérature selon la perspective actionnelle. Que pourrait être une « tâche littéraire »? " Le français à l'université, 14, $n^{\circ} 2$. Disponible en ligne. http://eprints.aidenligne-francais-universite.auf.org/137/.

Morel, A.-S. (2012). « Littérature et FLE : états des lieux, nouveaux enjeux et perspectives ». In Borg, S., (dir.). Synergies monde. Actes du IIème Forum Mondial HERACLES. Université de FrancheComté, $n^{\circ}$ 9, pp. 141-148.

Ouvrard, E. (2013). « Didactisation d'un document authentique en classe de langues : l'exemple de l'album de jeunesse en anglais ». Journée d'études « Le document pour l'enseignement et l'apprentissage : quelles questions aujourd'hui ? ». IUFM de Caen.

Puren, C. (2006). « Explication de textes et perspective actionnelle : la littérature entre le dire scolaire et le faire social ». Disponible en ligne. http://www.aplv-languesmodernes.org/spip.php? article389.

Puren, C. (2012). « Perspectives actionnelles sur la littérature dans l'enseignement scolaire et universitaire des langues-cultures : des tâches scolaires sur les textes aux actions sociales par les textes ». Disponible en ligne. http://www.christianpuren.com/mes-travaux-liste-et-liens/2012d/.

Saint-Gelais, R. (1999). « La Fiction à travers l'intertexte ». Colloque en ligne « Frontières de la fiction ». http://www.fabula.org/forum/colloque99/224.php.

Saint-Gelais, R. (2011). Atelier littéraire « Fictions transfuges ", Introduction à Fictions transfuges. La transfictionnalité et ses enjeux. Paris : Editions du Seuil, collection «Poétique ». pp. 7-17. Disponible en ligne. http://www.fabula.org/atelier.php?Fictions_transfuges.

Saint-Gelais, R. (2012) interviewé par Wagner, F., In « Fictions transfuges. La transfictionnalité et ses enjeux. Entretien avec Richard Saint-Gelais ». Vox Poetica. p. 1. Disponible en ligne. http:// www.vox-poetica.org/entretiens/intStGelais.html.

Van der Linden, S. (2006). Lire l'album. Le Puy-en-Velay : L'atelier du poisson soluble.

Zarate, G. (2003). Médiation culturelle et didactique des langues. Centre européen pour les langues vivantes. Strasbourg : Conseil de l'Europe.

\section{NOTES}

1. Ici le français, langue de l'école; je reprends ici une terminologie classique adoptée entre autres par Marie-Christine Deyrich et Suzanne Olive (2009).

2. Les documents d'accompagnement des programmes présentant des déclinaisons culturelles et linguistiques pour le cycle 2 et le cycle 3 sur Eduscol proposent de nombreuses utilisations d'albums de littérature de jeunesse.

3. Cette définition englobe les suites et continuations de tout genre et les cycles et séries. 
4. Dans l'enquête en ligne proposée par le laboratoire I3DL de l'université de Nice Sophia Antipolis, sur 5077 personnes interrogées (ayant lu au moins trois ouvrages de l'ensemble des Harry Potter et répondu à la question sur l'écriture de fanfictions), 1414, soit 27,9\%, avaient produit des écrits.

5. Personnage de sorcière créée par Helen Nicoll et Jan Pienkowsky.

6. Petit chien dont les aventures proposent un jeu de cache-cache à son jeune lecteur.

7. Nous pouvons citer par exemple Brown Bear, Brown Bear, What Do You See?, Polar Bear, Polar Bear, What Do You Hear? et Panda Bear, Panda Bear, What do You See?

8. http://www.ac-clermont.fr/dsden63/personnels/ressources-pedagogiques/enseignementsdisciplinaires/langues-vivantes/maternelle/.

\section{RÉSUMÉS}

Cet article s'intéresse à l'utilisation de l'album de littérature de jeunesse en cours de langues vivantes étrangères. S'il permet à l'élève d'acquérir des connaissances lexicales, grammaticales et phonologiques, il l'aide également à construire une compétence interculturelle : il est alors doublement médiateur. La question qui se pose ensuite est de savoir s'il peut être véritablement exploité en tant que support littéraire. Un détour, par la recherche en littérature et l'étude des pratiques transfictionnelles, nous permettra de définir les relations en jeu entre le texte et son lecteur et de voir s'il est possible de jouer sur ces échanges à l'école. Les projets de "réécritures » des histoires, mis en place en classe de langue dans le cadre de la perspective actionnelle, ne permettent-ils pas à l'élève de devenir à son tour médiateur?

This article deals with the exploitation of picture-books in second language classes. As well as allowing pupils to acquire lexical, grammatical and phonological knowledge, they also help them build an intercultural competence: picture-books are therefore mediative in two ways. The question is whether those books can really be exploited as literary texts. A look at the work of researchers in literature and transfictionality will help us analyse what relationships are at stake between books and their readers and see if these relationships can be exploited at school. Can "rewriting" stories, as is done in a second language class in an action-oriented approach, enable pupils in turn to become mediators?

\section{INDEX}

Mots-clés : album, transfictionnalité, réécriture, médiation

Keywords : picture-books, transfictionality, rewriting, mediation

\section{AUTEUR}

\section{MARIE-FRANCE BURGAIN}

ESPE d'Aquitaine, LAB E3D - EA 7441

Maître de Conférences en anglais, ayant rédigé une thèse en littérature, Marie-France Burgain, enseigne sur le site de Pau de l'ESPE d'Aquitaine depuis septembre 2010. Elle y assure des cours 
auprès d'étudiants inscrits en master MEEF $1^{\text {ère }}$ et $2^{\text {ème }}$ années mention $1^{\text {er }}$ degré mais aussi, en partenariat avec l'Université de Pau et des Pays de l'Adour, à des étudiants inscrits en master MEEF anglais. Elle s'intéresse à la didactique de l'anglais et, plus généralement, à la didactique des langues et cultures et, tout particulièrement, aux liens entre littérature de jeunesse et enseignement/apprentissage des langues.

marie.burgain[at]u-bordeaux.fr

LAB E3D - EA 7441 - ESPE d'Aquitaine

Site de Pau - 44, Bd Jean Sarrailh - 64000 Pau 